\title{
Incidence and Demographics of Nasopharyngeal Carcinoma in Cheung Chau Island of Hong Kong - A Distinct Geographical area with Minimal Residential Mobility and Restricted Public Healthcare Referral Network
}

\section{Sik-Kwan Chan}

University of Hong Kong

Sze-Chun Chau

University of Hong Kong

Sum-Yin Chan

University of Hong Kong

Chi-Chung Tong

University of Hong Kong

Ka-On Lam

University of Hong Kong

\section{Dora Kwong}

University of Hong Kong

\section{To-Wai Leung}

University of Hong Kong

Mai-Yee Luk

University of Hong Kong

\section{Anne Lee}

University of Hong Kong

Horace Choi

University of Hong Kong

Victor Lee ( $\nabla$ victorhflee@yahoo.com.hk)

University of Hong Kong

\section{Research Article}

Keywords: Nasopharyngeal carcinoma, Incidence, Mortality, Cheung Chau Island, Hong Kong

Posted Date: January 20th, 2021

DOI: https://doi.org/10.21203/rs.3.rs-146785/v1

License: (1) This work is licensed under a Creative Commons Attribution 4.0 International License. Read Full License 


\section{Abstract}

Background: Nasopharyngeal carcinoma (NPC) is endemic in Hong Kong with a skewed geographical and ethnic distribution. We performed an epidemiological study of NPC in Cheung Chau Island, a fishing village with very minimal residential mobility, and compared its demographics and survival with the rest of Hong Kong.

Methods: NPC data in Cheung Chau and non-Cheung Chau residents between 2006 and 2017 treated in our tertiary center were collected. The incidence, stage distribution and mortality of Cheung Chau NPC residents were compared with those of their counterparts in the whole Hong Kong obtained from the Hong Kong Cancer Registry. Propensity score matching (PSM) was performed between Cheung Chau and non-Cheung Chau cases in a 1:4 ratio. Overall Survival (OS), progression-free survival (PFS), and cancer-specific survival (CSS) were compared between these two cohorts by product limit estimation and log-rank tests.

Results: Sixty-one patients residing in Cheung Chau were identified between 2006 and 2017. There was a significantly higher NPC incidence $(P<0.001)$ but insignificant difference in mortality rate in Cheung Chau compared to the whole Hong Kong data. After PSM with 237 non-Cheung Chau patients, Cheung Chau cohort revealed a stronger NPC family history $(P<0.001)$. However, there were no significant differences in OS (P=0.170), PFS $(P=0.053)$, and CSS $(P=0.160)$ between these two cohorts.

Conclusion: Our results revealed that Cheung Chau had a higher NPC incidence but similar survival outcomes compared to the whole Hong Kong. Cheung Chau can provide an excellent model for further studies on NPC oncogenesis and screening.

\section{Background}

The epidemiology of nasopharyngeal carcinoma (NPC) is unique for its distinctly skewed geographical distribution [1]. It is rare in most parts of the world but it is endemic in Southeast Asia and southern China including Hong Kong. In 2018, it was the sixth commonest cancer in men and the seventeenth in women in Hong Kong and accounted for $2.4 \%$ of all new cancer cases. Extensive epidemiological studies of NPC in endemic areas have been carried out for the past few decades [2-8].

The public healthcare referral system in Hong Kong is divided into seven geographical clusters, namely Hong Kong West, Hong Kong East, Kowloon West, Kowloon Central, Kowloon East, New Territories West and New Territories East, under the management of the Hospital Authority, the only statutory public healthcare provider serving the whole Hong Kong population of more than 7.5 million living in an area of $1107 \mathrm{~km}^{2}$. Cheung Chau Island, one of the largest outlying islands in Hong Kong, is located about $10 \mathrm{~km}$ southwest and connected to Hong Kong Island only by pubic ferries where the central business district, the major governmental offices and residential areas are situated (Fig. 1). Cheung Chau has a comparably large population of over 22000 people with an area of $2.46 \mathrm{~km}^{2}$, making it the most densely populated island in Hong Kong (8519 persons $/ \mathrm{km}^{2}$ compared to 6788 persons $/ \mathrm{km}^{2}$ in the whole Hong Kong territory) [9]. Although Cheung Chau belongs to the Hong Kong East Cluster (HKEC) in terms of public healthcare provision (mainly because of the availability of helicopter transport service for emergency cases), almost all residents in Cheung Chau who have cancers are referred to the Hong Kong West Cluster (HKWC) (because of geographical vicinity) in which Queen Mary Hospital (i.e. our tertiary referral center) is the only tertiary public oncology center as well as one of the two university teaching hospitals in Hong Kong for further management, if they wish to seek further management in the public sector [10]. On the other hand, there are only 6 private clinics and one public hospital (which only provides general internal medicine service) in Cheung Chau, the corresponding doctor-to-population ratio is 1:3493 which is significantly lower than that of the rest of Hong Kong (1:511), suggesting that Cheung Chau residents have a lower accessibility to healthcare services which may pose a risk of delayed cancer diagnosis and its subsequent management [11]. More importantly, Cheung Chau share some similarities with other island countries in Southeast Asia in which the majority of the indigenous inhabitants are Hakka and Hokkien Chinese fishermen and their descendants, who have the habit of preserving their catch into salted fish for later consumption. Their children also share 9the same dietary habits with their parents who consume salted fish at an early age. Furthermore, the residential and geographical mobility in Cheung Chau has remained stable (7.10\% in 2011 and 6.99\% in 2016 in Cheung Chau vs. 18.61\% in 2011 and 17.08\% in 2016 in the rest of Hong Kong), providing a good source of epidemiological studies on genetically-related diseases and cancers including NPC $[12,13]$.

Hong Kong Cancer Registry (HKCaR) is a population-based cancer registry, which covers essential data including incidence, mortality as well as distribution of age, sex and stage of major cancer types including NPC. However, such information is just confined to the 
whole Hong Kong territory, and corresponding data in among different administrative districts/regions within Hong Kong are not captured and provided. We hypothesized that Cheung Chau, in view of the aforementioned reasons, has a much higher incidence of NPC compared to the whole Hong Kong territory. Therefore, we carried out this study to investigate the NPC incidence, mortality as well as its stage distribution and survival outcomes in this island which were compared to the figures in the whole Hong Kong territory, including all NPC patients treated in the era of intensity-modulated radiation therapy between 2006 and 2017. The results shall facilitate us to carry out future studies on genetic and dietary predisposing factors as well as highly focused screening and preventive measures for this prevalent malignancy within the highly endemic sub-district(s) of Hong Kong.

\section{Methods}

\section{Patient selection}

The population data of Cheung Chau in the official census and by-census years in Hong Kong, i.e. 2006, 2011 and 2016 were obtained from the Hong Kong Census and Statistics Department while those in the remaining years were estimated by interpolation based on the census data [12-14]. The incidence and mortality rates of NPC in Hong Kong between 2006 and 2017 were retrieved from HKCaR [15], while the corresponding data of NPC patients living in Cheung Chau as well as their NPC treatment details in the same time period were identified from Department of Clinical Oncology of Queen Mary Hospital in HKWC (i.e. our tertiary referral center) as previously mentioned. All the data obtained from Hong Kong Census and Statistics Department are freely available to the general public with the need for prior approval. In the current study, the TNM stage of every patient's previously untreated NPC was re-staged in accordance with 8th edition of AJCC/UICC TNM staging system for further stage distribution comparison between the Cheung Chau cohort, non-Cheung Chau cohort treated in our tertiary referral center and all the NPC cases registered in HKCaR between 2006 and 2017. Approval from the local institutional review board for this study was obtained before study commencement (Institutional Review Board of the University of Hong Kong/Hospital Authority Hong Kong West Cluster, reference number UW 12153 and UW 06-242 T/1267). It was conducted according to the guidelines of Declaration of Helsinki and Good Clinical Practice.

\section{Statistical analysis}

The crude incidence and mortality rates of NPC in Cheung Chau during this period were calculated and their differences against the whole Hong Kong territory were subsequently demonstrated in odds ratio (OR). The rates were then standardized by the direct method using the world standard population as the reference. Poisson regression model was employed to estimate the average annual percentage change (AAPC) of the standardized rates so as to measure the temporal trend of NPC. AAPC with a $95 \%$ confidence interval $(\mathrm{Cl})$ of $>0$ was considered a significantly increasing trend while AAPC with a $95 \% \mathrm{Cl}$ of $<0$ was considered a significant decreasing trend.

To further compare the survivor outcomes between Cheung Chau and non-Cheung Chau patients treated our tertiary referral center, we used propensity score matching (PSM) approach to adjust for unbalanced population and potential baseline confounders. A logistic regression model was established to calculate the propensity scores in which age, sex, T-category, N-category, and Mcategory were considered as predefined matching covariates. Patients living in Cheung Chau were matched with those living in regions other than Cheung Chau (the non-Cheung Chau cohort) but all treated in our tertiary referral center on a basis of nearest neighbor matching at a 1:4 ratio, in light of the huge population size difference between Cheung Chau and outside Cheung Chau.

Product-limit estimation of survival outcomes and log-rank tests were calculated for comparing survival differences between Cheung Chau and non-Cheung Chau patients before and after PSM. The survival endpoints in this study included progression-free survival (PFS), overall survival (OS) and cancer-specific survival (CSS).

All data analyses were performed by Statistical Package for the Social Sciences (SPSS) version 26.0. All tests were two-sided and $p$ values less than 0.05 were considered statistically significant.

\section{Results}

\section{Incidence and mortality}


Between 2006 and 2017, a total of 61 incident NPC cases, including 47 males and 14 females, in Cheung Chau were registered and treated in our tertiary referral center (Table 1), of them $96.7 \%$ had non-keratinizing undifferentiated carcinoma. The overall stage distribution of these 61 Cheung Chau NPC patients was: stage I in 1 (1.6\%); stage II in 8 (13.1\%); stage III in 27 (44.3\%); IVA in 19 (31.1\%); IVB in 6 (9.8\%). There was no significant difference in stage distribution between patients in Cheung Chau cohort and those in the whole Hong Kong territory obtained from HKCaR $(P=0.884)$. However, Cheung Chau cohort had a stronger family history of NPC, before $(29.5 \%$ vs $10.6 \%, P<0.001)$ and after PSM $(29.5 \%$ vs. $8.0 \%, P<0.001)$ (Table 1$)$. 
Table 1

Baseline patient characteristics before and after propensity score matching

\begin{tabular}{|c|c|c|c|c|c|c|c|c|}
\hline \multirow[b]{3}{*}{ Variables } & \multicolumn{8}{|c|}{ Patients, No. (\%) } \\
\hline & \multicolumn{4}{|c|}{ Before propensity score matching } & \multicolumn{4}{|c|}{ After propensity score matching } \\
\hline & $\begin{array}{l}\text { Total } \\
(916)\end{array}$ & $\begin{array}{l}\text { Cheung } \\
\text { Chau } \\
(61)\end{array}$ & $\begin{array}{l}\text { Non- } \\
\text { Cheung } \\
\text { Chau } \\
(855)\end{array}$ & $\mathrm{p}$ & $\begin{array}{l}\text { Total } \\
\text { (298) }\end{array}$ & $\begin{array}{l}\text { Cheung } \\
\text { Chau } \\
(61)\end{array}$ & $\begin{array}{l}\text { Non- } \\
\text { Cheung } \\
\text { Chau } \\
(237)\end{array}$ & $\mathrm{p}$ \\
\hline Median age in years (Range) & $\begin{array}{l}51.5(11- \\
90)\end{array}$ & $\begin{array}{l}49(11- \\
79)\end{array}$ & $52(16-90)$ & 0.851 & $\begin{array}{l}50(11- \\
85)\end{array}$ & $\begin{array}{l}49 \\
(11- \\
79)\end{array}$ & $\begin{array}{l}51(16- \\
85)\end{array}$ & 0.711 \\
\hline Male/female & $\begin{array}{l}686(74.9) \\
/ 230 \\
(25.1)\end{array}$ & $\begin{array}{l}47(77) \\
/ 14 \\
(23)\end{array}$ & $\begin{array}{l}639(74.3) \\
/ 216 \\
(25.7)\end{array}$ & 0.687 & $\begin{array}{l}229 \\
(76.8) / \\
69(23.2)\end{array}$ & $\begin{array}{l}47(77) \\
/ 14 \\
(23)\end{array}$ & $\begin{array}{l}182(76.8) \\
/ 55 \\
(23.2)\end{array}$ & 0.966 \\
\hline Family history of NPC & 109 (11.9) & $\begin{array}{l}18 \\
(29.5)\end{array}$ & $91(10.6)$ & $\begin{array}{l}< \\
0.001\end{array}$ & $37(12.4)$ & $\begin{array}{l}18 \\
(29.5)\end{array}$ & $19(8.0)$ & $\begin{array}{l}<.001 \\
0.001\end{array}$ \\
\hline Smoker/ ex-smoker & $336(36.7)$ & $\begin{array}{l}16 \\
(26.2)\end{array}$ & $320(37.4)$ & 0.080 & $\begin{array}{l}109 \\
(36.6)\end{array}$ & $\begin{array}{l}16 \\
(26.2)\end{array}$ & $93(39.2)$ & 0.068 \\
\hline Drinker & $300(32.8)$ & $\begin{array}{l}21 \\
(34.4)\end{array}$ & $279(32.6)$ & 0.852 & $97(32.6)$ & $\begin{array}{l}21 \\
(34.4)\end{array}$ & $76(32.1)$ & 0.895 \\
\hline Types of carcinoma & & & & 0.603 & & & & 0.578 \\
\hline Keratinizing & $3(0.3)$ & $0(0)$ & $3(0.7)$ & & $2(0.7)$ & $0(0)$ & $2(0.8)$ & \\
\hline Non-keratinizing differentiated & $16(1.7)$ & $2(3.3)$ & $14(1.7)$ & & $6(2.0)$ & $2(3.3)$ & $4(1.8)$ & \\
\hline $\begin{array}{l}\text { Non-keratinizing } \\
\text { undifferentiated }\end{array}$ & 897 (97.9) & $\begin{array}{l}59 \\
(96.7)\end{array}$ & $838(98.0)$ & & $\begin{array}{l}291 \\
(97.7)\end{array}$ & $\begin{array}{l}59 \\
(96.7)\end{array}$ & $232(98.1)$ & \\
\hline ECOG performance status & & & & 0.061 & & & & 0.105 \\
\hline 0 & 91 (13.7) & $\begin{array}{l}11 \\
(18.0)\end{array}$ & $250(29.2)$ & & $78(26.2)$ & $\begin{array}{l}11 \\
(18.0)\end{array}$ & $67(28.3)$ & \\
\hline $1-2$ & $655(71.5)$ & $\begin{array}{l}50 \\
(82.0)\end{array}$ & 605 (70.7) & & $\begin{array}{l}220 \\
(73.8)\end{array}$ & $\begin{array}{l}50 \\
(82.0)\end{array}$ & 170 (71.7) & \\
\hline T-category & & & & 0.042 & & & & 0.884 \\
\hline T1 & $222(24.2)$ & 7 (11.5) & $215(25.1)$ & & $32(10.5)$ & $\begin{array}{l}7 \\
(11.5)\end{array}$ & $25(10.5)$ & \\
\hline T2 & 206 (22.5) & $\begin{array}{l}15 \\
(24.6)\end{array}$ & $191(22.3)$ & & $64(21.5)$ & $\begin{array}{l}15 \\
(24.6)\end{array}$ & $49(20.7)$ & \\
\hline T3 & $370(40.4)$ & $\begin{array}{l}26 \\
(42.6)\end{array}$ & $344(40.2)$ & & $\begin{array}{l}139 \\
(46.6)\end{array}$ & $\begin{array}{l}26 \\
(42.6)\end{array}$ & 113 (47.7) & \\
\hline T4 & 118 (12.9) & $\begin{array}{l}13 \\
(21.3)\end{array}$ & 105 (12.3) & & $63(21.1)$ & $\begin{array}{l}13 \\
(21.3)\end{array}$ & $50(21.1)$ & \\
\hline $\mathrm{N}$-category & & & & 0.071 & & & & 0.864 \\
\hline NO & $132(14.4)$ & $4(6.6)$ & $128(15.0)$ & & $23(7.7)$ & $4(6.6)$ & $19(9.0)$ & \\
\hline N1 & $212(24.8)$ & $\begin{array}{l}13 \\
(21.3)\end{array}$ & $212(24.8)$ & & 57 (19.1) & $\begin{array}{l}13 \\
(21.3)\end{array}$ & $44(18.6)$ & \\
\hline N2 & 328 (38.4) & $\begin{array}{l}33 \\
(54.1)\end{array}$ & $328(38.4)$ & & $\begin{array}{l}155 \\
(52.0)\end{array}$ & $\begin{array}{l}33 \\
(54.1)\end{array}$ & $122(51.5)$ & \\
\hline
\end{tabular}




\begin{tabular}{|c|c|c|c|c|c|c|c|c|}
\hline \multirow[b]{2}{*}{ N3 } & \multicolumn{8}{|c|}{ Patients, No. (\%) } \\
\hline & $187(21.9)$ & $\begin{array}{l}11 \\
(18.0)\end{array}$ & $187(21.9)$ & & $63(21.1)$ & $11(18)$ & $52(21.9)$ & \\
\hline M-category & & & & 0.176 & & & & 0.487 \\
\hline MO & $862(94.1)$ & $\begin{array}{l}55 \\
(90.2)\end{array}$ & $807(94.4)$ & & $\begin{array}{l}275 \\
(92.3)\end{array}$ & $\begin{array}{l}55 \\
(90.2)\end{array}$ & $220(92.8)$ & \\
\hline M1 & $54(8.1)$ & $6(9.8)$ & $48(8.0)$ & & $23(7.7)$ & $6(9.8)$ & $17(7.2)$ & \\
\hline Overall stage & & & & 0.373 & & & & 0.884 \\
\hline I & $51(5.6)$ & $1(1.6)$ & $50(5.8)$ & & $4(1.3)$ & $1(1.6)$ & $3(1.3)$ & \\
\hline II & $152(16.6)$ & $8(13.1)$ & $144(16.8)$ & & $32(10.7)$ & $\begin{array}{l}8 \\
(13.1)\end{array}$ & $24(10.1)$ & \\
\hline III & $403(42.7)$ & $\begin{array}{l}27 \\
(44.3)\end{array}$ & $376(44.0)$ & & $\begin{array}{l}137 \\
(46.0)\end{array}$ & $\begin{array}{l}27 \\
(44.3)\end{array}$ & $110(46.4)$ & \\
\hline IVA & $256(27.9)$ & $\begin{array}{l}19 \\
(31.1)\end{array}$ & $237(27.7)$ & & $\begin{array}{l}102 \\
(34.2)\end{array}$ & $\begin{array}{l}19 \\
(31.1)\end{array}$ & $83(35.0)$ & \\
\hline IVB & $54(5.9)$ & $6(9.8)$ & $48(5.6)$ & & $23(7.7)$ & $6(9.8)$ & $17(7.2)$ & \\
\hline Radical IMRT only & $81(8.8)$ & $4(6.6)$ & $77(9.0)$ & 0.515 & $28(9.4)$ & $4(6.6)$ & $24(10.2)$ & 0.483 \\
\hline $\begin{array}{l}\text { Concurrent chemoradiation } \\
\text { alone }\end{array}$ & $320(40.9)$ & $\begin{array}{l}21 \\
(34.4)\end{array}$ & $299(40.8)$ & 0.798 & $\begin{array}{l}113 \\
(37.9)\end{array}$ & $\begin{array}{l}21 \\
(34.4)\end{array}$ & $92(38.8)$ & 0.863 \\
\hline $\begin{array}{l}\text { Induction chemotherapy then } \\
\text { concurrent chemoradiation }\end{array}$ & $203(28.2)$ & $\begin{array}{l}18 \\
(29.5)\end{array}$ & $203(23.7)$ & 0.309 & $89(29.9)$ & $\begin{array}{l}18 \\
(29.5)\end{array}$ & $71(30.0)$ & 0.895 \\
\hline $\begin{array}{l}\text { Concurrent chemoradiation } \\
\text { then adjuvant chemotherapy }\end{array}$ & $260(33.2)$ & $\begin{array}{l}13 \\
(21.3)\end{array}$ & $247(33.7)$ & 0.205 & $79(26.5)$ & $\begin{array}{l}13 \\
(21.3)\end{array}$ & $66(27.8)$ & 0.302 \\
\hline Palliative chemotherapy & $94(10.3)$ & $6(9.8)$ & 88 (10.3) & 0.910 & $36(12.1)$ & $6(9.8)$ & 30 (12.7) & 0.546 \\
\hline $\begin{array}{l}\text { Consolidation RT after } \\
\text { palliative chemotherapy }\end{array}$ & $53(5.8)$ & $6(9.8)$ & 47 (5.5) & 0.161 & $24(8.1)$ & $6(9.8)$ & $18(7.6)$ & 0.566 \\
\hline
\end{tabular}

The crude incidence rate of NPC in Cheung Chau over 2006-2017 was 22.6 per 100,000 population (95\% Cl: 17.3-29.0). Table 2 shows incidence rate by age groups. There was no significant change observed in NPC trend in Cheung Chau (AAPC: $+13.5 \%$; $95 \%$ $\mathrm{Cl}$ : $-37.6 \%$ - +64.6\%). In the same period, there were 10,447 new cases of NPC in the whole Hong Kong territory and the crude incidence was 12.2 per 100,000 population. The odds of getting NPC were $85 \%$ higher in Cheung Chau than those in the whole Hong Kong territory (OR:1.85; 1.42-2.38; $P<0.001)$. The age-standardized incidence rate was 7.9 per 100,000 population and a significant decreasing trend was observed (AAPC: $-2.6 \% ; 95 \% \mathrm{Cl}:-5.0 \%--0.3 \%$ ) for the whole Hong Kong territory. The age-standardized rate of NPC in Cheung Chau doubled that in the whole Hong Kong territory $(P<0.001)$. 
Table 2

Age standardized incidence rates (per 100,000 persons) of nasopharyngeal carcinoma by year and age group in Cheung Chau and in whole Hong Kong territory

\begin{tabular}{|lllllll|}
\hline \multicolumn{3}{|c|}{ Age group (years) } & \multicolumn{3}{c|}{ Age standardized incidence } \\
\hline Year & $0-19$ & $20-44$ & $45-64$ & $65+$ & Cheung Chau (vs whole Hong Kong territory) \\
\hline $2006-2007$ & 10.1 & 22.2 & 29.6 & 44.0 & $20.3(9.2)$ \\
\hline $2008-2009$ & 0 & 5.6 & 60.8 & 14.6 & $14.5(8.7)$ \\
\hline $2010-2011$ & 0 & 16.9 & 39.2 & 0 & $13.2(7.9)$ \\
\hline $2012-2013$ & 0 & 17.8 & 8.0 & 0 & $7.6(7.4)$ \\
\hline $2014-2015$ & 16.2 & 25.5 & 40.3 & 23.0 & $24.4(7.4)$ \\
\hline $2016-2017$ & 0 & 6.8 & 98.7 & 21.4 & $22.6(6.9)$ \\
\hline Overall & & & & & \\
\hline $2006-2017$ & 4.3 & 15.8 & 45.7 & 17.3 & $17.0(7.9)$ \\
\hline
\end{tabular}

There were 17 NPC deaths in the Cheung Chau cohort and its crude mortality rate was 6.3 per 100,000 population (95\% Cl: $3.7-10.1)$ over 2006-2017. On the other hand, NPC caused 3,983 deaths in the whole Hong Kong territory in the same period with the crude mortality of 4.7 per 100,000 population. There is no significant difference in NPC mortality between Cheung Chau and the whole Hong Kong territory (OR: 1.34; $95 \% \mathrm{Cl}: 0.78-2.14 ; P=0.23$ ). The age standardized mortality rate of NPC of the Cheung Chau cohort and the whole Hong Kong territory were 5.0 and 2.8 respectively per 100,000 population (Table 3 ). No significant change in mortality over 2006-2017 was observed in both the Cheung Chau cohort (AAPC: $+18.4 \% ; 95 \% \mathrm{Cl}:-49.9 \%-+81.6 \%$ ) and the whole Hong Kong territory (AAPC: $-4.0 \%$; $95 \% \mathrm{Cl}:-9.7 \%-+1.7 \%$ ).

Table 3

Age standardized mortality rates (per 100,000 persons) of nasopharyngeal carcinoma by year and age group in Cheung Chau and in whole Hong Kong territory

\begin{tabular}{|llllll|}
\hline \multicolumn{3}{|c}{ Age group (years) } & \multicolumn{2}{l|}{ Age standardized mortality } \\
\hline Year & $0-19$ & $20-44$ & $45-64$ & $65+$ & Cheung Chau (vs whole Hong Kong territory) \\
\hline $2006-2007$ & 0 & 0 & 0 & 0 & $0(3.4)$ \\
\hline $2008-2009$ & 0 & 0 & 15.2 & 14.6 & $3.9(3.2)$ \\
\hline $2010-2011$ & 0 & 5.6 & 7.8 & 14.6 & $4.4(2.9)$ \\
\hline $2012-2013$ & 0 & 5.9 & 8.0 & 13.0 & $4.4(2.6)$ \\
\hline $2014-2015$ & 16.2 & 0 & 32.3 & 0 & $12.6(2.5)$ \\
\hline $2016-2017$ & 0.0 & 0 & 32.9 & 0 & $6.3(2.4)$ \\
\hline Overall & & & & & \\
\hline $2006-2017$ & 2.2 & 2.0 & 15.7 & 6.5 & $5.0(2.8)$ \\
\hline
\end{tabular}

\section{Survival outcomes before PSM}

The 61 NPC patients in the Cheung Chau cohort had marginally worse survival outcomes than the non-Cheung Chau counterparts: 5year PFS $(60.3 \%$ vs. $70.3 \%, P=0.033)$, OS $(70.8 \%$ vs $82.4 \%, P=0.109)$ and CSS $(73.2 \%$ vs. $86.6 \%, P=0.064)$ (Supplemental Fig. 1 and Table 4). When comparing the survival by stage, their survival outcomes (PFS, OS and CSS) were also not different from nonCheung Chau patients, except for stage I disease. 
Table 4

Survival endpoints of nasopharyngeal carcinoma patients stratified by their residence and disease stage before and after propensity score matching

Before propensity score matching

Progression- Overall Survival

free Survival

5 -year $\quad 95 \% \quad 5$-year $\quad 95 \% \mathrm{Cl}$

rate $\mathrm{Cl}$ rate

\section{After propensity score matching}

\section{Cancer-specific Survival}

5-year $\quad 95 \%$ rate $\mathrm{Cl}$
Progression-
free Survival

5-year 95\% rate
Overall Surviva

Cancer-specific Survival

5-year 95\% 5-year 95\%

rate $\mathrm{Cl}$ rate $\mathrm{Cl}$

\section{Overall study population}

\begin{tabular}{|c|c|c|c|c|c|c|c|c|c|c|c|c|}
\hline $\begin{array}{l}\text { Cheung } \\
\text { Chau }\end{array}$ & $60.3 \%$ & $\begin{array}{l}47.1- \\
73.6 \%\end{array}$ & $70.8 \%$ & $57.8-83.8 \%$ & $73.2 \%$ & $\begin{array}{l}60.2- \\
86.2 \%\end{array}$ & $60.3 \%$ & $\begin{array}{l}47.1- \\
73.6 \%\end{array}$ & $70.8 \%$ & $\begin{array}{l}57.8- \\
83.8 \%\end{array}$ & $73.2 \%$ & $\begin{array}{l}60.2- \\
86.2 \%\end{array}$ \\
\hline $\begin{array}{l}\text { Non- } \\
\text { Cheung } \\
\text { Chau }\end{array}$ & $70.3 \%$ & $\begin{array}{l}67.0- \\
73.6 \%\end{array}$ & $82.4 \%$ & $79.7-85.1 \%$ & $86.6 \%$ & $\begin{array}{l}84.1- \\
89.0 \%\end{array}$ & $69.2 \%$ & $\begin{array}{l}62.9- \\
75.5 \%\end{array}$ & $81.8 \%$ & $\begin{array}{l}76.4- \\
87.1 \%\end{array}$ & $86.0 \%$ & $\begin{array}{l}81.2- \\
90.8 \%\end{array}$ \\
\hline$p$ & 0.033 & & 0.109 & & 0.064 & & 0.053 & & 0.170 & & 0.160 & \\
\hline
\end{tabular}

\section{Stage I}

\begin{tabular}{|c|c|c|c|c|c|c|c|c|c|c|c|c|}
\hline $\begin{array}{l}\text { Cheung } \\
\text { Chau }\end{array}$ & $0 \%$ & $0-0 \%$ & $0 \%$ & $0-0 \%$ & $0 \%$ & $0-0 \%$ & $0 \%$ & $0-0 \%$ & $0 \%$ & $0-0 \%$ & $0 \%$ & $0-0 \%$ \\
\hline $\begin{array}{l}\text { Non- } \\
\text { Cheung } \\
\text { Chau }\end{array}$ & $91.2 \%$ & $\begin{array}{l}83.8- \\
100 \%\end{array}$ & $93.5 \%$ & $86.1-100 \%$ & $100 \%$ & $\begin{array}{l}100- \\
100 \%\end{array}$ & $100 \%$ & $\begin{array}{l}100- \\
100 \%\end{array}$ & $100 \%$ & $\begin{array}{l}100- \\
100 \%\end{array}$ & $100 \%$ & $\begin{array}{l}100- \\
100 \%\end{array}$ \\
\hline$p$ & $<0.001$ & & $<0.001$ & & $<0.001$ & & 0.083 & & 0.083 & & 0.083 & \\
\hline \multicolumn{13}{|l|}{ Stage II } \\
\hline $\begin{array}{l}\text { Cheung } \\
\text { Chau }\end{array}$ & $37.5 \%$ & $\begin{array}{l}24.6- \\
96.1 \%\end{array}$ & $75.0 \%$ & $50.0-100 \%$ & $100 \%$ & $\begin{array}{l}60.6- \\
100 \%\end{array}$ & $37.5 \%$ & $\begin{array}{l}24.4- \\
96.2 \%\end{array}$ & $75.0 \%$ & $\begin{array}{l}45.5- \\
100 \%\end{array}$ & $100 \%$ & $\begin{array}{l}60.6- \\
100 \%\end{array}$ \\
\hline $\begin{array}{l}\text { Non- } \\
\text { Cheung } \\
\text { Chau }\end{array}$ & $80.7 \%$ & $\begin{array}{l}74.4- \\
88.3 \%\end{array}$ & $89.7 \%$ & $84.3-95.0 \%$ & $95.4 \%$ & $\begin{array}{l}92.1- \\
99.4 \%\end{array}$ & $87.5 \%$ & $\begin{array}{l}74.6- \\
100 \%\end{array}$ & $90.5 \%$ & $\begin{array}{l}65.2- \\
100 \%\end{array}$ & $85.7 \%$ & $\begin{array}{l}82.1- \\
98.6 \%\end{array}$ \\
\hline$p$ & 0.107 & & 0.018 & & 0.096 & & 0.072 & & 0.058 & & 0.162 & \\
\hline \multicolumn{13}{|l|}{ Stage III } \\
\hline $\begin{array}{l}\text { Cheung } \\
\text { Chau }\end{array}$ & $88.9 \%$ & $\begin{array}{l}89.4- \\
94.1 \%\end{array}$ & $100 \%$ & $72.4-100 \%$ & $86.3 \%$ & $\begin{array}{l}72.4- \\
100 \%\end{array}$ & $88.9 \%$ & $\begin{array}{l}67.8- \\
96.2 \%\end{array}$ & $100 \%$ & $\begin{array}{l}72.2- \\
100 \%\end{array}$ & $86.3 \%$ & $\begin{array}{l}72.6- \\
100 \%\end{array}$ \\
\hline $\begin{array}{l}\text { Non- } \\
\text { Cheung } \\
\text { Chau }\end{array}$ & $75.7 \%$ & $\begin{array}{l}71.2- \\
80.5 \%\end{array}$ & $87.4 \%$ & $84.4-91.6 \%$ & $92.6 \%$ & $\begin{array}{l}89.3- \\
96.4 \%\end{array}$ & $74.5 \%$ & $\begin{array}{l}66.1- \\
83.6 \%\end{array}$ & $82.9 \%$ & $\begin{array}{l}75.6- \\
91.2 \%\end{array}$ & $90.1 \%$ & $\begin{array}{l}84.2- \\
96.1 \%\end{array}$ \\
\hline$p$ & 0.991 & & 0.692 & & 0.982 & & 0.909 & & 0.635 & & 0.927 & \\
\hline
\end{tabular}

\section{Stage IVA}

\begin{tabular}{|c|c|c|c|c|c|c|c|c|c|c|c|c|}
\hline $\begin{array}{l}\text { Cheung } \\
\text { Chau }\end{array}$ & $61.4 \%$ & $\begin{array}{l}25.2- \\
72.1 \%\end{array}$ & $81.8 \%$ & $30.5-85.1 \%$ & $81.8 \%$ & $\begin{array}{l}30.8- \\
85.1 \%\end{array}$ & $61.4 \%$ & $\begin{array}{l}25.8- \\
72.0 \%\end{array}$ & $81.8 \%$ & $\begin{array}{l}42.4- \\
92.5 \%\end{array}$ & $81.8 \%$ & $\begin{array}{l}30.8- \\
85.1 \%\end{array}$ \\
\hline $\begin{array}{l}\text { Non- } \\
\text { Cheung } \\
\text { Chau }\end{array}$ & $63.6 \%$ & $\begin{array}{l}57.8- \\
70.3 \%\end{array}$ & $78.8 \%$ & $73.4-84.0 \%$ & $82.1 \%$ & $\begin{array}{l}77.6- \\
87.5 \%\end{array}$ & $65.8 \%$ & $\begin{array}{l}55.5- \\
77.6 \%\end{array}$ & $83.7 \%$ & $\begin{array}{l}75.3- \\
92.8 \%\end{array}$ & $85.0 \%$ & $\begin{array}{l}77.8- \\
93.2 \%\end{array}$ \\
\hline$p$ & 0.156 & & 0.469 & & 0.181 & & 0.119 & & 0.286 & & 0.237 & \\
\hline
\end{tabular}

95\% Cl: 95\% confidence interval. 


\begin{tabular}{|c|c|c|c|c|c|c|c|c|c|c|c|c|}
\hline \multirow[b]{2}{*}{$\begin{array}{l}\text { Cheung } \\
\text { Chau }\end{array}$} & \multicolumn{6}{|c|}{ Before propensity score matching } & \multicolumn{6}{|c|}{ After propensity score matching } \\
\hline & $20.0 \%$ & $\begin{array}{l}0- \\
71.1 \%\end{array}$ & $40.0 \%$ & $10.8 \%-90.1 \%$ & $60.0 \%$ & $\begin{array}{l}17.4- \\
100 \%\end{array}$ & $20.0 \%$ & $\begin{array}{l}0- \\
71.3 \%\end{array}$ & $40.0 \%$ & $\begin{array}{l}10.4- \\
90.4 \%\end{array}$ & $60.0 \%$ & $\begin{array}{l}17.4- \\
100 \%\end{array}$ \\
\hline $\begin{array}{l}\text { Non- } \\
\text { Cheung } \\
\text { Chau }\end{array}$ & $4.7 \%$ & $\begin{array}{l}0- \\
13.2 \%\end{array}$ & $18.1 \%$ & $4.6-32.2 \%$ & $18.1 \%$ & $\begin{array}{l}4.6- \\
32.1 \%\end{array}$ & $14.7 \%$ & $\begin{array}{l}0- \\
38.1 \%\end{array}$ & $37.3 \%$ & $\begin{array}{l}29.2- \\
100 \%\end{array}$ & $37.3 \%$ & $\begin{array}{l}12.4- \\
63.1 \%\end{array}$ \\
\hline$p$ & 0.033 & & 0.242 & & 0.111 & & 0.256 & & 0.692 & & 0.367 & \\
\hline
\end{tabular}

\section{Survival outcomes after PSM}

PSM was performed to adjust for potentially confounding variables including age, gender, T-category, N-category and M-category. A cohort of 237 non-Cheung Chau patients was matched. Table 1 shows the details of the variables before and after PSM, which revealed that the impact of potential selection bias was reduced.

Figure 2 and Table 4 show the survival differences between Cheung Chau patients and the matched cohort of non-Cheung Chau patients after PSM. There was no statistically significant difference in OS (70.8\% vs. $81.8 \%, P=0.170)$ (Fig. $2 \mathrm{~b})$ and CSS (73.2\% vs. $86.0 \%, P=0.160$ ) (Fig. 2c) between Cheung Chau and non-Cheung Chau patients, though there was a trend of worse PFS in Cheung Chau patients $(60.3 \%$ vs. $69.2 \%, P=0.053)$ (Fig. 2a). Similarly, significant differences in survival outcomes were not observed when stratified by stage.

\section{Discussion}

A progressive decline in both the incidence and the mortality of NPC was observed in Hong Kong over the period of 2006-2017 based on the data from HKCaR. Such improvement was most likely attributed to dietary modification, easier accessibility to tertiary public healthcare service, advances in diagnostic and surveillance tools as well as advent of multimodality and multidisciplinary treatment [16-21]. It is within our expectation that Cheung Chau showed no difference in NPC mortality rates and survival outcomes when compared to their counterparts in the whole Hong Kong population, since almost all NPC patients living in Cheung Chau received the same and prompt standard treatment based on standardized treatment protocols as for other NPC patients in all tertiary public oncology hospitals in Hong Kong under the management of the Hospital Authority, in which NPC management is one of the key performance indicators [22]. Besides, NPC management in Hong Kong has long been spearheaded by Hong Kong Nasopharyngeal Cancer Study Group established since 1993, composed of all NPC experts working in all public healthcare clusters in Hong Kong who have collaborated and published numerous clinical trials on NPC for the past 3 decades.

Cheung Chau population, on the other hand, is $85 \%$ more likely to develop NPC as compared to the whole Hong Kong population. Significant stronger family history of NPC in Cheung Chau patients was observed in our study. In view of geographical characteristics and the restricted geographical mobility of its residents, Cheung Chau is an ideal place to study NPC epidemiology for four main reasons. First, due to the limited access to public and private healthcare resources, most of the Cheung Chau patients will be referred to the single institution (i.e. our hospital) for further management. This provides convenient data collection and the data collected represent the unique characteristics of NPC in Cheung Chau. Second, most of the Cheung Chau residents are indigenous southern Chinese or Hakka and Hokkien descendants, who have a higher risk of developing NPC compared to other Asian ethnic groups [7,23]. The higher NPC incidence can provide more effective population size due to higher occupation rate. Third, salted fish is one of the major food consumed by Cheung Chau residents as they share similar dietary habits of their ancestors who were mostly fishermen. The correlation between Chinese-style salted fish consumption and NPC development has been proven and confirmed [24-27]. Finally, stable and restricted geographical mobility in Cheung Chau with such a small living area reduces the chance of case loss or case mix through emigration and immigration respectively. This strongly enables us to study the familial penetrance and inheritance patterns of NPC in greater details. 
Studies have suggested that childhood consumption of salted fish and other preserved food is one of the major risk factors of NPC among Chinese [24-32]. Risk of NPC was found to be increased with an earlier age of first exposure, frequency and duration of consumption of these food items. Cheung Chau has traditionally been an island of fisher folk whose diet contains a high proportion of salted fish and others like preserved vegetables and shrimp paste. These are also common food consumed by inhabitants in Southern China, emigrant Chinese populations in Malaysia and other Southeast Asian populations [26, 28]. All of these population groups have intermediate to high susceptibility to NPC development as demonstrated in previous studies $[21,26,28]$. We believe that this traditional southern Chinese dietary factor still plays an important role in developing NPC in Cheung Chau at present in spite of decreasing proportion of fishery workers and the adoption of more westernized diet nowadays. In addition, incense burning, a traditional daily practice in Chinese households, is associated with an increased NPC risk. Cheung Chau is a well-known place with lots of Chinese temples organizing large-scale annual Chinese folk religious activities which involve frequent incense burning [33, 34]. Prolonged inhalation of carcinogens from incense burning was shown to significantly increase the risk of having NPC [34, 35].

We acknowledge several limitations in our study. First, we could not provide a clear and representative record of the amount of salted fish consumption and exposure to incense burning of our Cheung Chau patients owing to the retrospective nature of this study. Furthermore, there was a possibility of not collecting Cheung Chau NPC cases managed at other public or private hospitals. Having said that, we believe that we have captured almost all Cheung Chau patients diagnosed with NPC in Hong Kong within the study period since our institution is the only public oncology hospital and tertiary referral center in closest proximity to Cheung Chau, which welcomes and provides comprehensive cancer service to all Cheung Chau patients. However, any extra Cheung Chau NPC cases treated outside our institution would imply even a higher incidence than that presented in the current study. Closer collaboration with other hospitals and private practice would certainly enhance the robustness of our data.

\section{Conclusions}

Cheung Chau, a geographically and ethnically distinct place, shows a higher than average NPC incidence and a stronger family history of NPC when compared to the rest of Hong Kong. It provides an ideal condition for future research in NPC carcinogenesis, screening, and prevention.

\section{Abbreviations}

NPC: nasopharyngeal carcinoma; HKEC: Hong Kong East Cluster; HKCaR: Hong Kong Cancer Registry; AJCC/UICC: American Joint Committee on Cancer/Union for International Cancer Control; TNM: tumour, node, metastasis; OR: odds ratio; AAPC: average annual percentage change; $95 \% \mathrm{Cl}$ : 95\% confidence interval; PSM: propensity score matching; PFS: progression-free survival; OS: overall survival; CSS: cancer-specific survival.

\section{Declarations}

\section{Ethics approval and consent to participate}

This study was approved by the Institutional Review Board of the University of Hong Kong/Hospital Authority Hong Kong West Cluster (reference number UW 12-153 and UW 06-242 T/1267). Informed consent was obtained from all subjects or, if subjects are under 16, from a parent and/or legal guardian. Access to the raw data from Hong Kong Census and Statistics Department does not require any prior administrative permissions. All methods were performed in accordance with Declaration of Helsinki and Good Clinical Practice.

\section{Consent for publication}

Not applicable.

\section{Availability of data and materials}


The datasets presented in this article are not readily available due to national legislation and the terms of the study's ethics approval do not allow dataset sharing outside of the institutions participating in the analysis. The corresponding author should be contacted for any reasonable request for any data of this study.

\section{Competing interest}

The authors declare that they have no competing interests.

\section{Funding}

The authors received no specific funding for this work.

\section{Authors' contributions}

SKC, SCC, AWML, HCWC and VHFL designed the study. SKC, SCC, HCWC and VHFL performed the statistical analysis. All authors contributed patient data and participated in reviewing and improving statistical analysis and manuscript. All authors read and approved the final manuscript.

\section{Acknowledgements}

The authors would like to thank all the staff members of Department of Clinical Oncology of Queen Mary Hospital, Hong Kong for their contributions.

\section{References}

1. Chen YP, Chan ATC, Le QT, Blanchard P, Sun Y, Ma J. Nasopharyngeal carcinoma. Lancet. 2019;394(10192):64-80.

2. Zhang F, Li W, Xie S, Ling W, Chen S, Liu Q, et al. Incidence trend of nasopharyngeal carcinoma from 1987 to 2011 in Sihui County, Guangdong Province, South China: an age-period-cohort analysis. Cancer Comm. 2015;34(3):1-8.

3. Wei K, Zhang R, Zhang S, Liang Z, Li Z, Chen W. Nasopharyngeal carcinoma incidence and mortality in China. Cancer Comm. 2017; 36(1):1-8.

4. Cammoun M, Moerner VG, Mourali N. Tumours of the nasopharynx in Tunisia. Cancer. 1974; 33(1):184-92.

5. Hirayama T. Descriptive and analytical epidemiology of nasopharyngeal cancer. IARC Sci Publ. 1978;(20):167-89.

6. Hidayatalla A, Malik MO, El Hadi AE, et al. Studies on nasopharyngeal carcinoma in the Sudan-I. Epidemiology and Aetiology. Eur J Cancer Clin Oncol. 1983;19(6):705-10.

7. Wee JT, Ha TC, Loong SL, Qian CN. Is nasopharyngeal cancer really a "Cantonese cancer"? Chin J Cancer. 2010;29(5):517-26.

8. Kataki AC, Simons MJ, Das AK, Sharma K, Mehra NK. Nasopharyngeal carcinoma in the Northeastern states of India. Chin J Cancer. 2011;30(2):106-13.

9. Census and Statistics Department. District Profiles. https://www.bycensus2016.gov.hk/en/bc-dp.html. Accessed 31 Dec 2020.

10. Hospital Authority. St. John Hospital. https://www.ha.org.hk/visitor/ha_visitor_text_index.asp? Content_ID=100146\&Lang=ENG\&Ver=TEXT. Accessed 31 Dec 2020.

11. The Medical Council of Hong Kong. List of Registered Medical Practitioners. https://www.mchk.org.hk/english/list_register/list.php?type=L. Accessed 31 Dec 2020.

12. Census and Statistics Department. 2011 Population Census. https://www.censtatd.gov.hk/hkstat/sub/so170.jsp. Accessed 31 Dec 2020.

13. Census and Statistics Department. 2016 Population By-census. https://www.censtatd.gov.hk/hkstat/sub/so459.jsp. Accessed 31 Dec 2020. 
14. Census and Statistics Department. 2006 Population By-census. https://www.censtatd.gov.hk/hkstat/sub/so440.jsp. Accessed 31 Dec 2020.

15. Hong Kong Cancer Registry, Hospital Authority. Cancer Facts. https://www3.ha.org.hk/cancereg/facts.html. Accessed 31 Dec 2020.

16. Lee AWM, Foo W, Law SCK, Poon YF, Sze WM, O SK, et al. Nasopharyngeal carcinoma: presenting symptoms and duration before diagnosis. Hong Kong Med J. 1997;3(4):355- 61.

17. Lee AWM, Poon YF, Foo W, Law SCK, Cheung FK, Chan DKK, et al. Retrospective analysis of 5037 patients with nasopharyngeal carcinoma treated during 1976 -1985: overall survival and patterns of failure. Int J Radiat Oncol Biol Phys. 1992;23(2):261-70.

18. Haberer-Guillerm S, Touboul E, Huguet F. Intensity modulated radiation therapy in nasopharyngeal carcinoma. Eur Ann Otorhinolaryngol Head Neck Dis. 2015;132(3):147-51.

19. Lee AW, Ma BB, Ng WT, Chan ATC. Management of nasopharyngeal carcinoma: current practice and future perspective. J Clin Oncol. 2015;33(29):3356-64.

20. Chua ML, Chan AT. Gemcitabine: a game changer in nasopharyngeal carcinoma. Lancet. 2016;388(10054):1853-4.

21. Greta C, Eva N, Daisuke K, Garavello W, Vecchia CL, Malvezzi M. Global trends in nasopharyngeal cancer mortality since 1970 and predictions for 2020: Focus on low-risk areas. Int J Cancer. 2017;140(10):2256-64.

22. Hospital Authority. Report on Key Performance Indicators. https://www.ha.org.hk/visitor/ha_visitor_text_index.asp? Content_ID=237981\&Lang=ENG\&Dimension=100\&Parent_ID=10011. Accessed 31 Dec 2020.

23. Chang ET, Adami HO. The enigmatic epidemiology of nasopharyngeal carcinoma. Cancer Epidemiol Biomarkers Prev. 2006;15(10):1765-77.

24. Ho JHC, Huang DP, Fong YY. Salted fish and nasopharyngeal carcinoma in southern Chinese. Lancet. 1978;2(8090):626.

25. Ho JHC. Genetic and environmental factors in nasopharyngeal carcinoma. In: Nakahara W, Nishioka K, Hirayama T, eds. Recent advances in human tumor virology and immunology. Tokyo: University of Tokyo Press 1971:275-95.

26. Armstrong RW, Armstrong MJ, Yu MC, Henderson B. Salted fish and inhalants as risk factors in nasopharyngeal carcinoma in Malaysian Chinese. Cancer Res. 1983;43(6):2967-70.

27. Huang DP, Ho JHC. Salted fish and nasopharyngeal carcinoma. In: Stich HF, ed. Carcinogen and mutagens in the environment naturally occurring compounds: epidemiology and distribution. Volume III. USA: CRC Press. 1983:21-7.

28. Poirier S, Ohshima H, de The G, Hubert A, Bourgade MC, Bartsch H. Volatile nitrosamine levels in common food from Tunisia, South China, and Greenland, high risk areas for nasopharyngeal carcinoma (NPC). Int J Cancer. 1987;39(3):292-6.

29. Yu MCC. Nasopharyngeal carcinoma: epidemiology and dietary factors. NUH-CME Rev. 1993;3:92-6.

30. Yuan JM, Wang XL, Xiang YB, Gao YT, Ross RK, Yu MC. Preserved foods in relation to risk of nasopharyngeal carcinoma in Shanghai, China. Int J Cancer. 2000;85(3):358-63.

31. Shivappa N, Hebert JR, Zucchetto A, Montella M, Libra M, Garavello W, et al. Increased risk of nasopharyngeal carcinoma with increasing levels of diet-associated inflammation in an Italian case-control study. Nutr Cancer. 2016;68(7):1123-30.

32. Yong SK, Ha TC, Yeo MCR, Gaborieay V, McKay JD, Wee J. Associations of lifestyle and diet with the risk of nasopharyngeal carcinoma in Singapore: a case-control study. Cancer Comm. 2017;36:3.

33. Cheung Chau Jiao Festival. The Representative List of the Intangible Cultural Heritage of Hong Kong. Intangible Cultural Heritage Office. The Government of the Hong Kong Special Administrative Region. https://www.Icsd.gov.hk/CE/Museum/ICHO/en_US/web/icho/representative_list_cheungchau.html. Accessed 31 Dec 2020.

34. Xie HS, Yu IT, Tse LA, Au JSK, Wang F, Lau JSM, et al. Domestic incense burning and nasopharyngeal carcinoma: a case-control study in Hong Kong Chinese. Environ Mol Mutagen. 2014;55(9):751-6.

35. He YQ, Xue WQ, Shen GP, Tang LL, Zeng YX, Jia WH. Household inhalants exposure and nasopharyngeal carcinoma risk: a large-scale case-control study in Guangdong, China. BMC Cancer. 2015;15:1022.

\section{Figures}



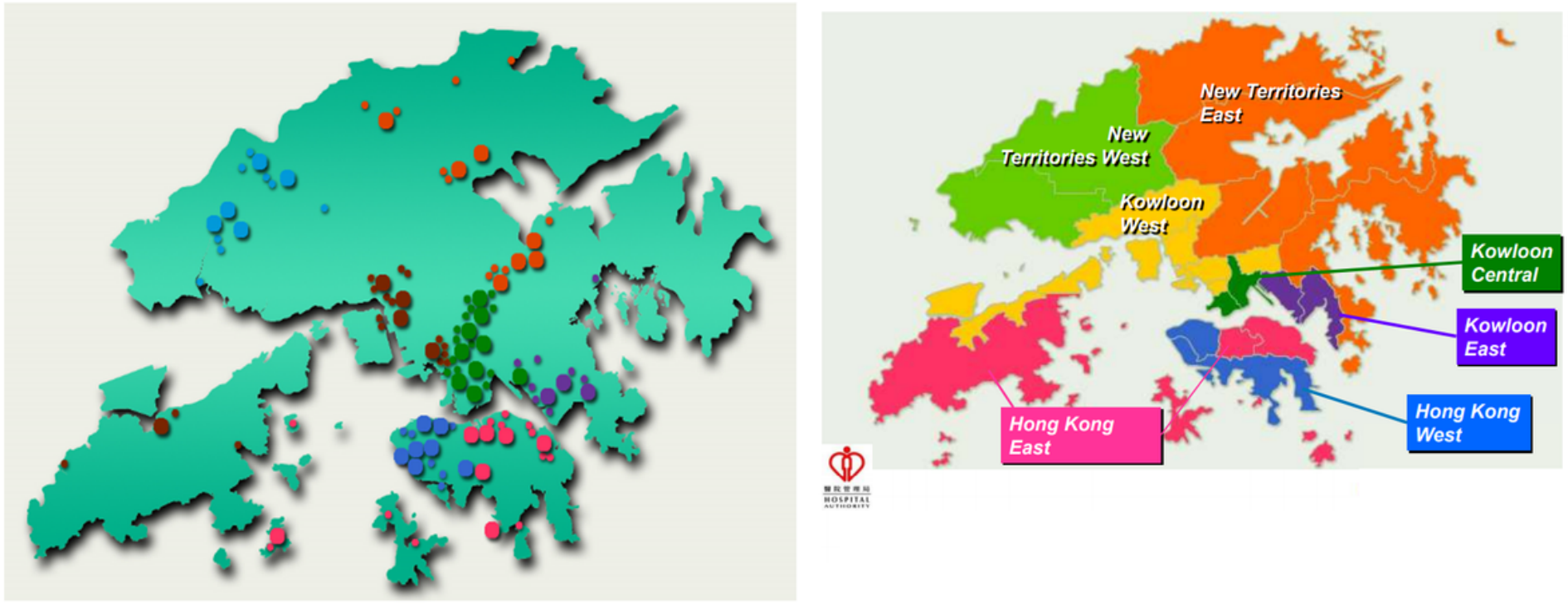

\section{Figure 1}

a Geographical location of Cheung Chau in Hong Kong. b geographical representations of seven clusters of public healthcare services under the management of Hospital Authority based on locations. Color dots in a represent the locations of government hospitals (larger dots) and their satellite out-patient clinics (smaller dots) within their respective clusters. Adapted and modified from Clusters, Hospitals \& Institutions. The Hospital Authority. https://www.ha.org.hk/visitor/template101.asp?

Parent_ID $=10004 \&$ Content_ID $=10036 \&$ Note: The designations employed and the presentation of the material on this map do not imply the expression of any opinion whatsoever on the part of Research Square concerning the legal status of any country, territory, city or area or of its authorities, or concerning the delimitation of its frontiers or boundaries. This map has been provided by the authors.
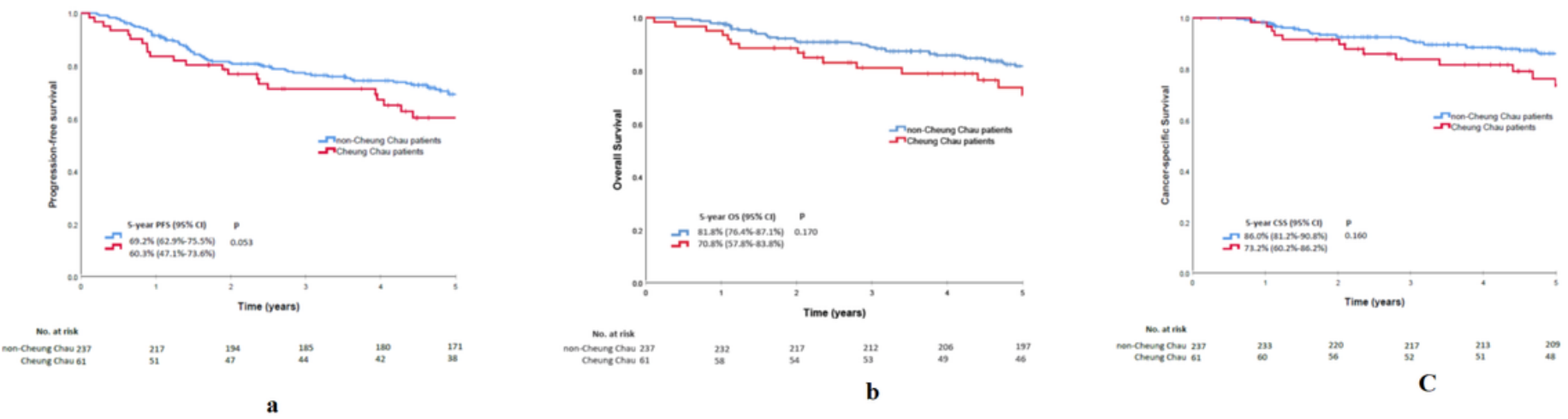

\section{Figure 2}

Kaplan-Meier estimations of survival outcomes of Cheung Chau patients and non-Cheung Chau patients with nasopharyngeal carcinoma after propensity score matching. a Progression-free survival. b overall survival. c cancer-specific survival

\section{Supplementary Files}

This is a list of supplementary files associated with this preprint. Click to download.

- SupplementaryMaterial.docx 